\title{
Unlocking Rapid Charging and Extended Life- Times for Li-Ion Batteries Using Freestanding Quantum Conversion-Type Aerofilm Anode
}

Sun-Sik Kim ${ }^{a, \#}$, Sung Mi Jung ${ }^{b, \#}$, Chenrayan Senthil ${ }^{a, *}$, and Hyun Young Jung ${ }^{a, c, *}$

${ }^{a}$ Department of Energy Engineering, Gyeongsang National University, Jinju-si, Gyeongnam 52725, South Korea.

${ }^{\mathrm{b}}$ Environmental Fate \& Exposure Research Group, Korea Institute of Toxicology, Jinju-si, Gyeongnam 52834, South Korea.

${ }^{\mathrm{c}}$ Future Convergence Technology Research Institute, Gyeongsang National University, Jinjusi, Gyeongnam 52725, South Korea.

\# Authors equally contributed

* To whom correspondence should be addressed. E-mail: csenthil@gnu.ac.kr (C. Senthil), hyjung@gnu.ac.kr (H.Y. Jung) 
Fig. S1. Radar chart comparison showing Li-ion diffusivity, tortuosity, resistance, activity, and energy and power densities for various $\mathrm{SnO}_{2}$ nanoparticles-based anode.

Fig. S2. Full XPS survey scan spectra depicted for various active nanoparticles in $\mathrm{SnO}_{2}$ anodes.

Fig. S3. XPS Spectra for different particle size of $\mathrm{SnO}_{2}$-based anode. a-c) $10 \mathrm{~nm}$, d-f) $100 \mathrm{~nm}$, and g-i) $200 \mathrm{~nm}$.

Fig. S4. Cyclic Voltammetry(CV) curves for $\mathrm{SnO}_{2}$ anodes using a) $10 \mathrm{~nm}$, b) $100 \mathrm{~nm}$, c) 200 $\mathrm{nm}$ active nanoparticles obtained at a scan rate of $1 \mathrm{mV} \mathrm{s}^{-1}$.

Fig. S5. Cyclic Voltammetry curves for $\mathrm{SnO}_{2}$ anodes using a-c) $10 \mathrm{~nm}$, d-f) $100 \mathrm{~nm}$ and g-i) $200 \mathrm{~nm}$ active nanoparticles obtained at various scan rate of $0.1,0.2,0.5,1.0 \mathrm{mV} \mathrm{s}^{-1}$.

Fig. S6. a) Power density for $\mathrm{SnO}_{2}$-based anodes calculated from the $\mathrm{CV}$ curve corresponding to $5^{\text {th }}$ Cycle at $1 \mathrm{mV} \mathrm{s}^{-1}$.

Fig. S7. a,c,e) Charge-discharge profiles and b,d,f) capacity contributions for $\mathrm{SnO}_{2}$ anodes using a-b) $10 \mathrm{~nm}, \mathrm{c}-\mathrm{d}) 100 \mathrm{~nm}$, e-f), $200 \mathrm{~nm}$ active nanoparticles. Charge-discharge profiles were obtained at a current rate of $0.875 \mathrm{~A} \mathrm{~g}^{-1}(1 \mathrm{C})$. Capacity contributions were analyzed by reconversion and dealloying reactions between the potential ranges $2.5-1.0 \mathrm{~V}$ and $1.0-0.05 \mathrm{~V}$.

Fig. S8. a) SEM micrographs at low- and high-magnification of physically mixing $\mathrm{CNT} / \mathrm{SnO}_{2}$ anode. b) Capacity comparison according to cycles at $1 \mathrm{C}$ of the physically mixing $\mathrm{CNT} / \mathrm{SnO}_{2}$ anode, $\mathrm{CNT} / \mathrm{SnO}_{2}$ anode using a binder, and quantum $\mathrm{SnO}_{2}$ anode with $\mathrm{Sn}-\mathrm{C}$ chemical bond.

Fig. S9. Electrochemical charge-discharge profile for the $3 \mathrm{~nm}$ quantum $\mathrm{SnO}_{2}$ anode at a) 10 $\mathrm{C}\left(8.75 \mathrm{~A} \mathrm{~g}^{-1}\right)$ and b) $20 \mathrm{C}\left(17 \mathrm{~A} \mathrm{~g}^{-1}\right)$ 
Fig. S10. Post-mortem analysis of cycled various particle size of $\mathrm{SnO}_{2}$-based anode through XRD and SAED pattern. a) $3 \mathrm{~nm}$, b) $10 \mathrm{~nm}$, and c) $100 \mathrm{~nm}$.

Fig. S11. EDS mapping image for quantum size of $\mathrm{SnO}_{2}$.

Table S1. Quantitaive values depicted in Fig. 1 b derived from various $\mathrm{SnO}_{2}$ anodes.

Table S2. EIS fitted parameters for $\mathrm{SnO}_{2}$-based anode using different active nanoparticles.

Table S3. Comparison of electrochemical performance of previously reported $\mathrm{SnO}_{2}$ based anodes for Li-ion batteries.

Table S4. Deconvoluted peak area obtained from the C1s and O1s spectra through XPS measurement. 


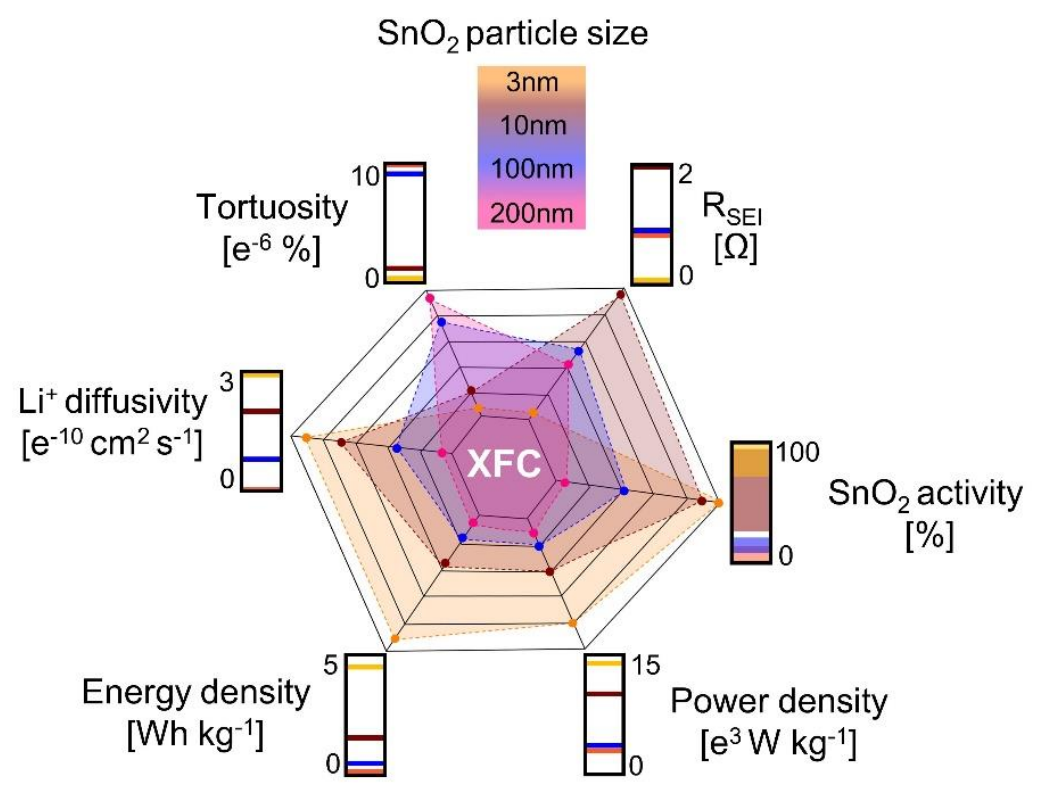

Fig. S1. Radar chart comparison showing Li-ion diffusivity, tortuosity, resistance, activity, and energy and power densities for various $\mathrm{SnO}_{2}$ nanoparticles-based anode.

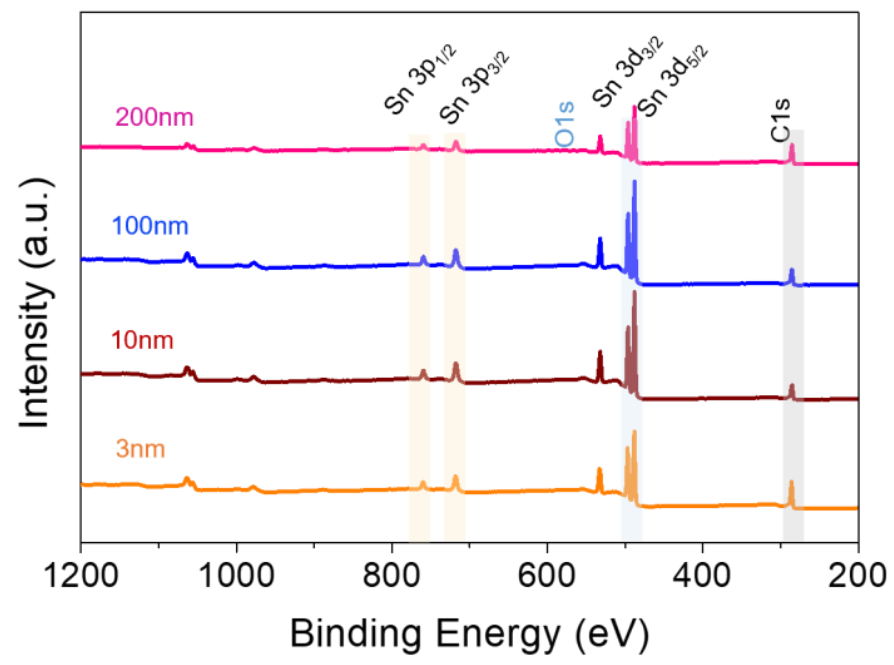

Fig. S2. Full XPS survey scan spectra depicted for various active nanoparticles in $\mathrm{SnO}_{2}$ anodes. 

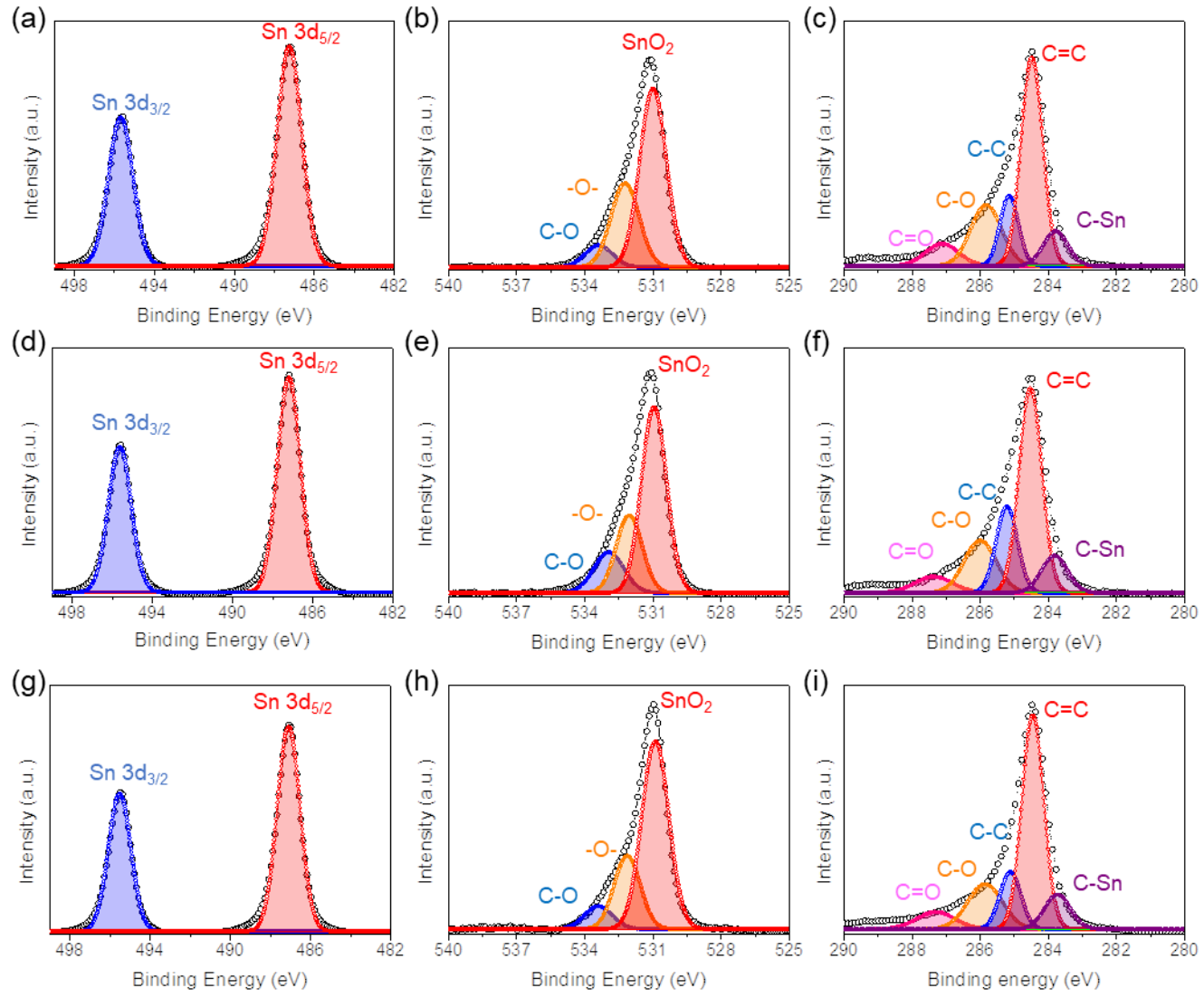

Fig. S3. XPS Spectra for different particle size of $\mathrm{SnO}_{2}$-based anode. a-c) $\left.10 \mathrm{~nm}, \mathrm{~d}-\mathrm{f}\right) 100 \mathrm{~nm}$, and g-i) $200 \mathrm{~nm}$. 

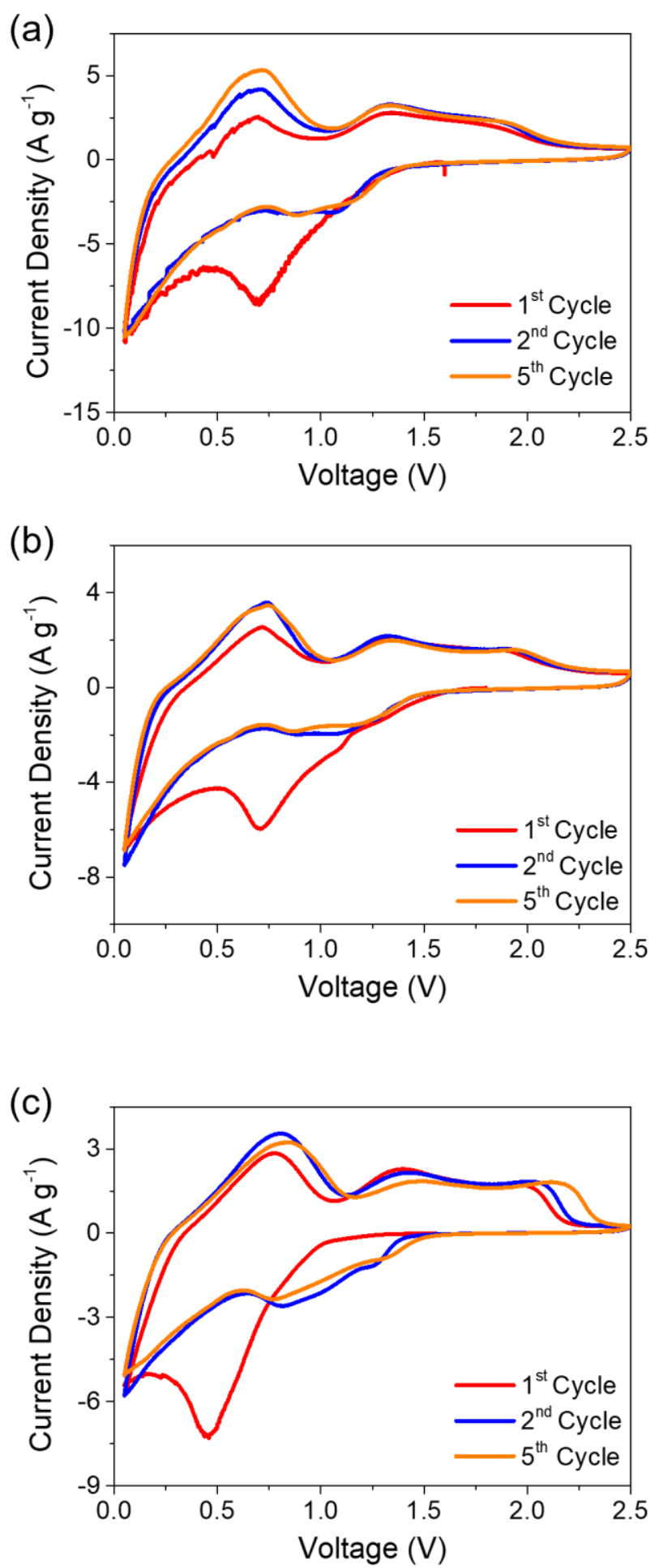

Fig. S4. Cyclic Voltammetry(CV) curves for $\mathrm{SnO}_{2}$ anodes using a) $10 \mathrm{~nm}$, b) $100 \mathrm{~nm}$, c) 200 $\mathrm{nm}$ active nanoparticles obtained at a scan rate of $1 \mathrm{mV} \mathrm{s}^{-1}$. 

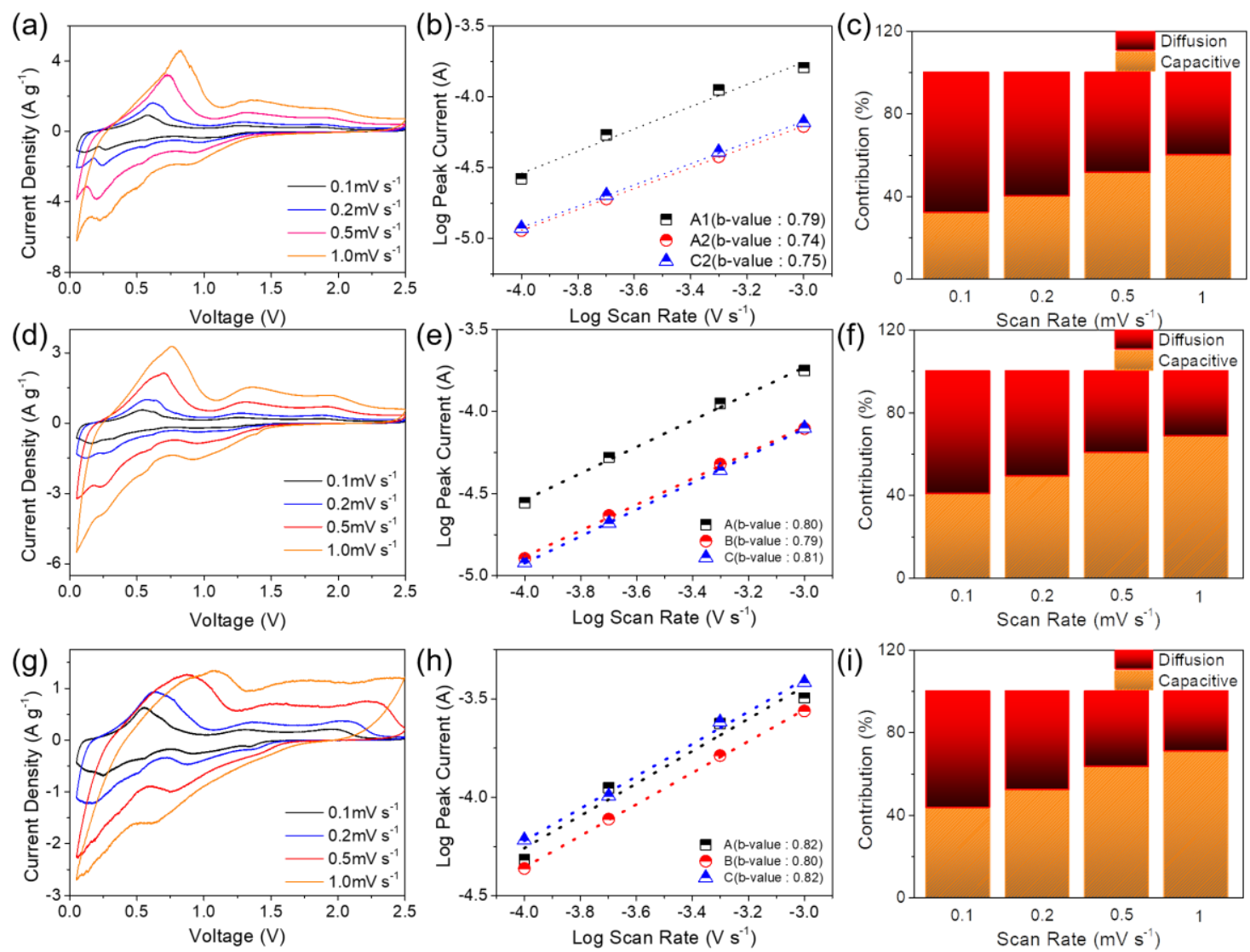

Fig. S5. Cyclic Voltammetry curves for $\mathrm{SnO}_{2}$ anodes using a-c) $10 \mathrm{~nm}$, d-f) $100 \mathrm{~nm}$ and g-i) $200 \mathrm{~nm}$ active nanoparticles obtained at various scan rate of $0.1,0.2,0.5,1.0 \mathrm{mV} \mathrm{s}^{-1}$. 


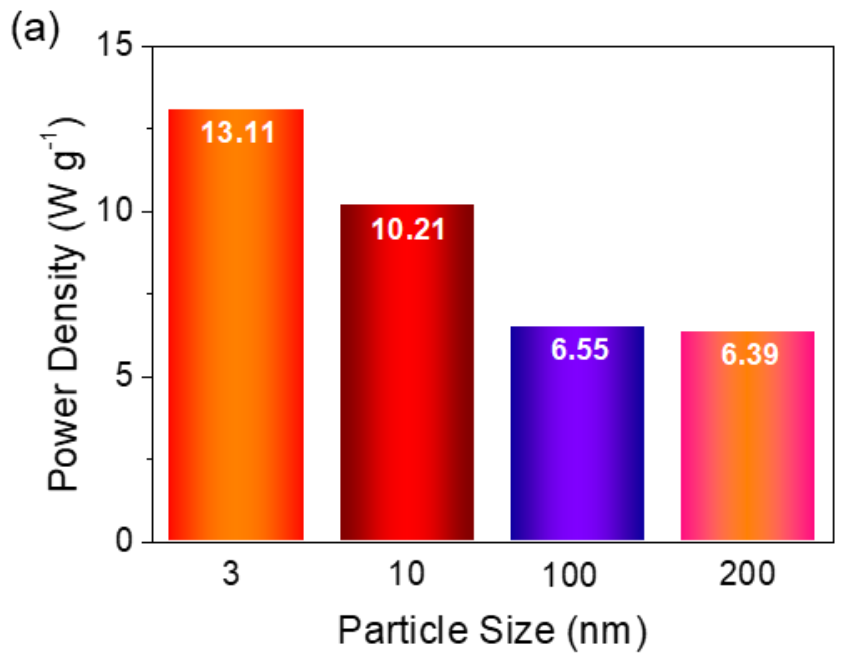

Fig. S6. a) Power density for $\mathrm{SnO}_{2}$-based anodes calculated from the CV curve corresponding to $5^{\text {th }}$ Cycle at $1 \mathrm{mV} \mathrm{s}^{-1}$. 


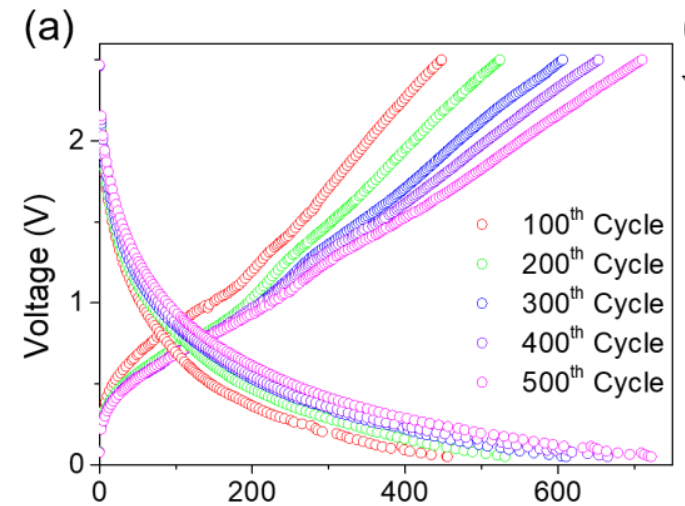

(c) Specific Capacity $\left(\mathrm{mAh} \mathrm{g}^{-1}\right)$

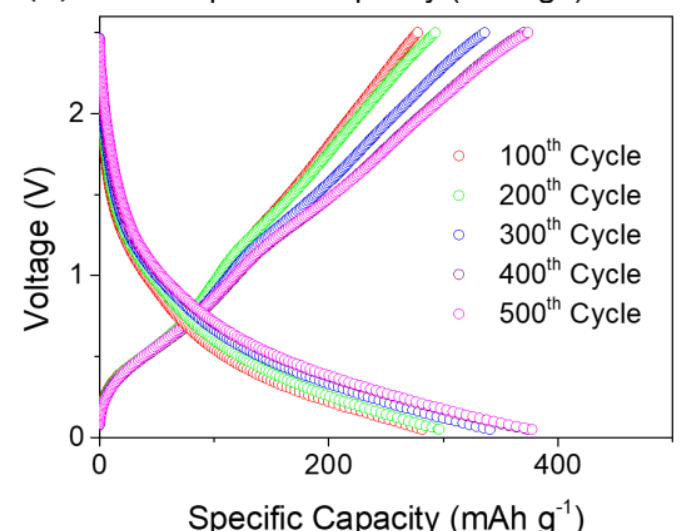

(e)

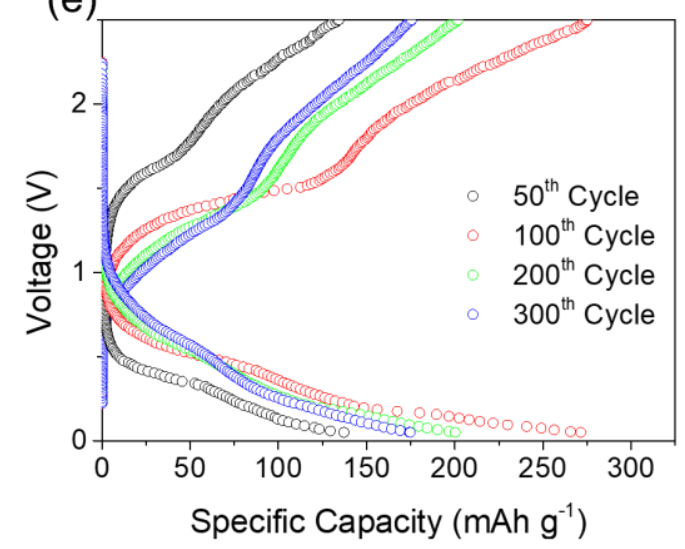

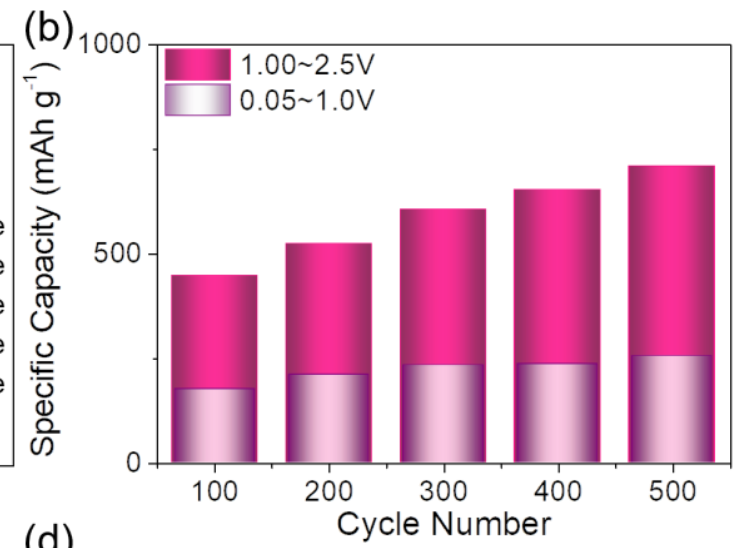

(d)

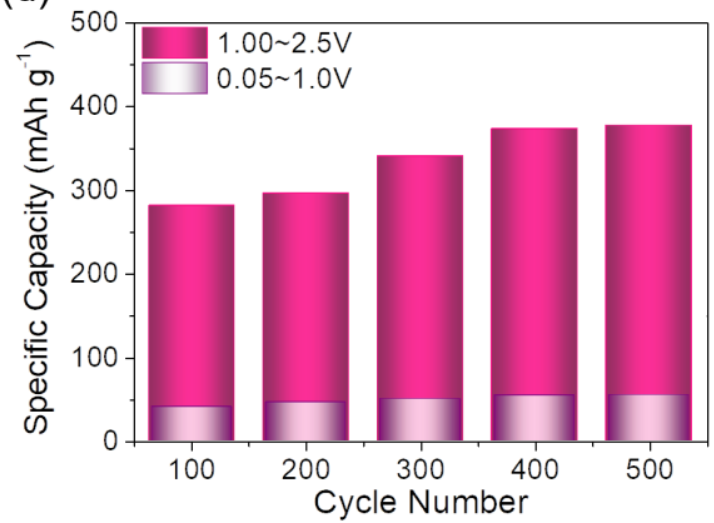

(f)

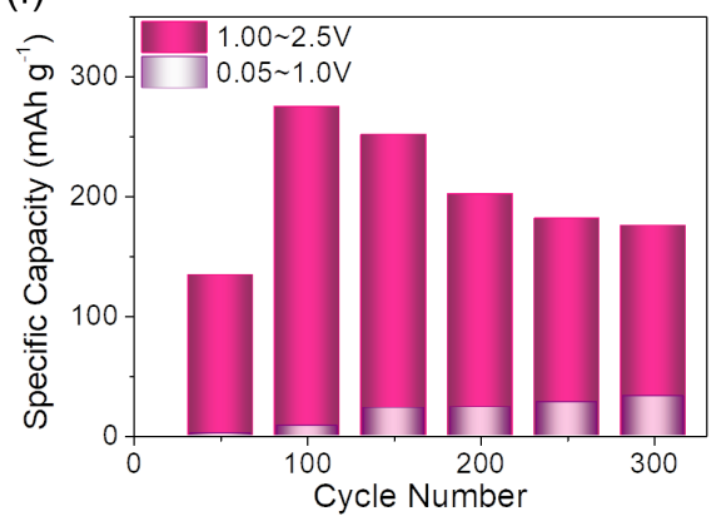

Fig. S7. a,c,e) Charge-discharge profiles and b,d,f) capacity contributions for $\mathrm{SnO}_{2}$ anodes using a-b) $10 \mathrm{~nm}, \mathrm{c}-\mathrm{d}) 100 \mathrm{~nm}$, e-f), $200 \mathrm{~nm}$ active nanoparticles. Charge-discharge profiles were obtained at a current rate of $0.875 \mathrm{~A} \mathrm{~g}^{-1}(1 \mathrm{C})$. Capacity contributions were analyzed by reconversion and dealloying reactions between the potential ranges $2.5-1.0 \mathrm{~V}$ and $1.0-0.05 \mathrm{~V}$. 
(a)
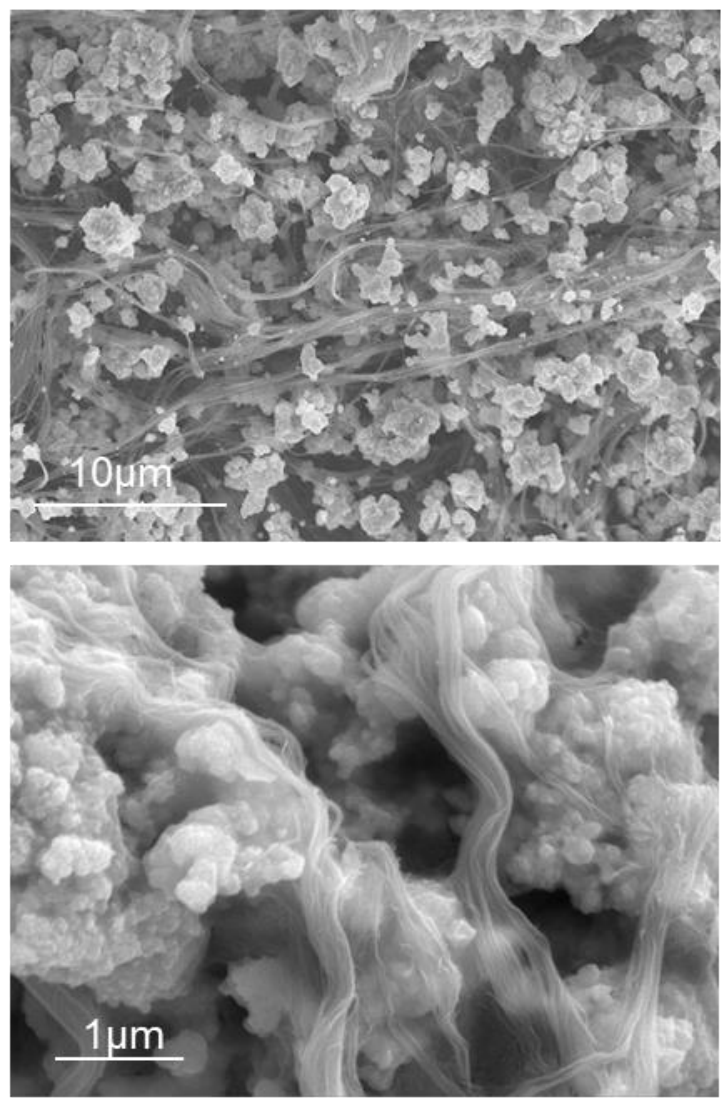

(b)

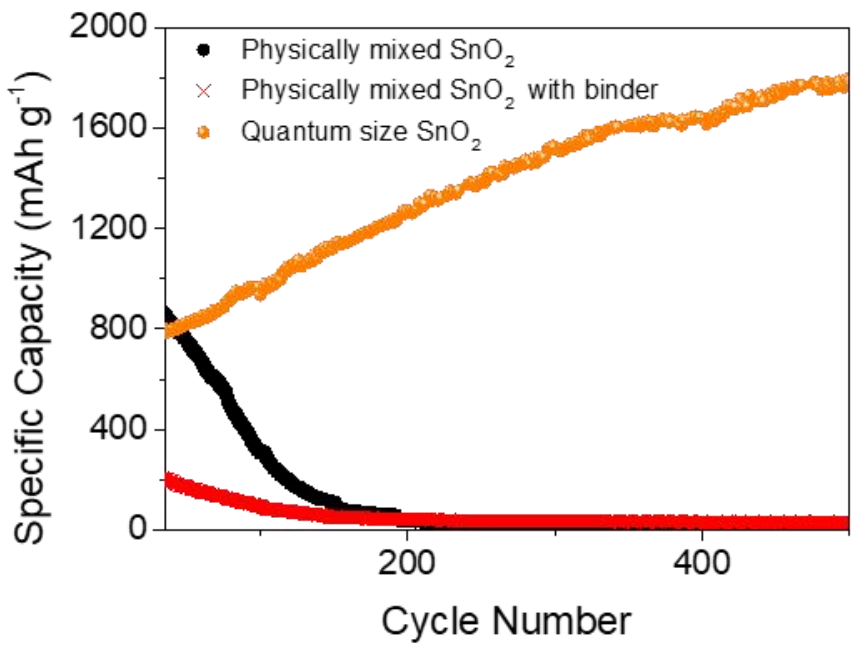

Fig. S8. a) SEM micrographs at low- and high-magnification of physically mixing $\mathrm{CNT} / \mathrm{SnO}_{2}$ anode. b) Capacity comparison according to cycles at $1 \mathrm{C}$ of the physically mixing $\mathrm{CNT} / \mathrm{SnO}_{2}$ anode, $\mathrm{CNT} / \mathrm{SnO}_{2}$ anode using a binder, and quantum $\mathrm{SnO}_{2}$ anode with $\mathrm{Sn}-\mathrm{C}$ chemical bond. 

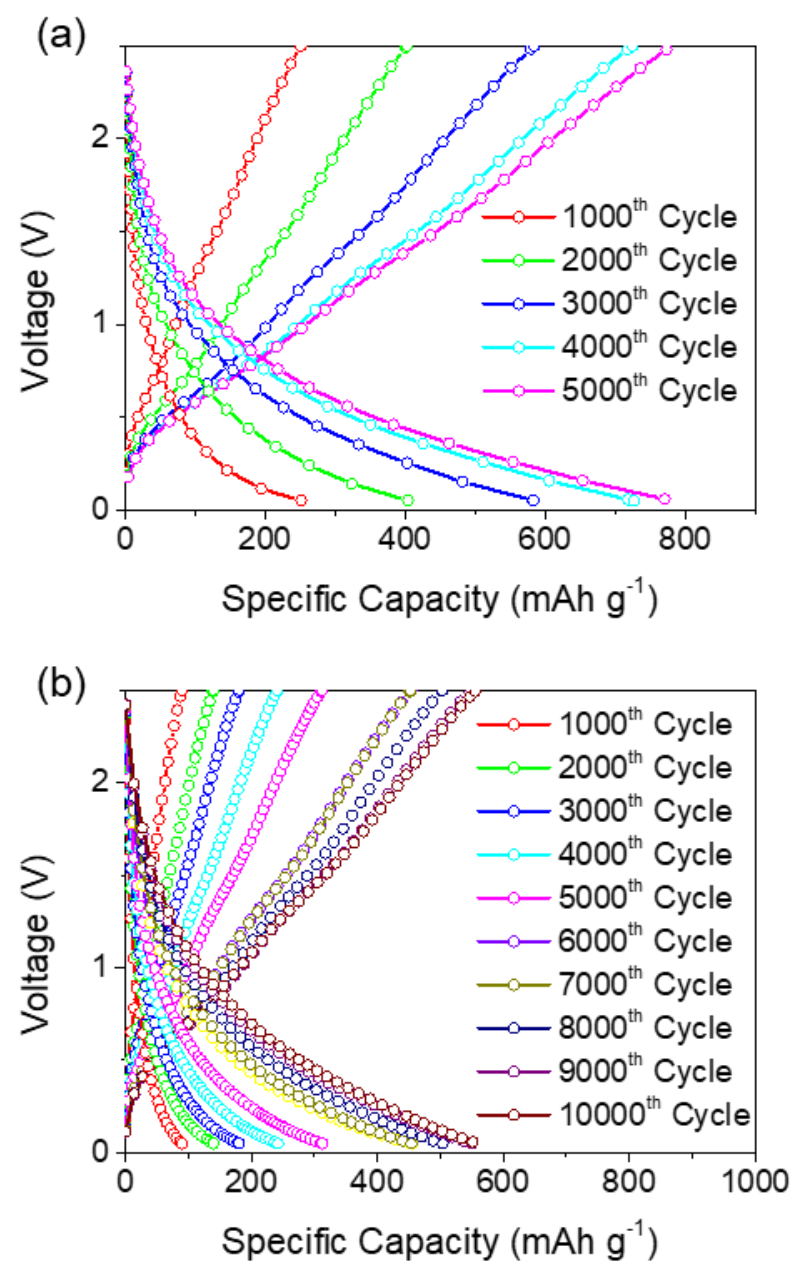

Fig. S9. Electrochemical charge-discharge profile for the $3 \mathrm{~nm}$ quantum $\mathrm{SnO}_{2}$ anode at a) 10 $\mathrm{C}\left(8.75 \mathrm{~A} \mathrm{~g}^{-1}\right)$ and b) $20 \mathrm{C}\left(17 \mathrm{~A} \mathrm{~g}^{-1}\right)$. 
(a)
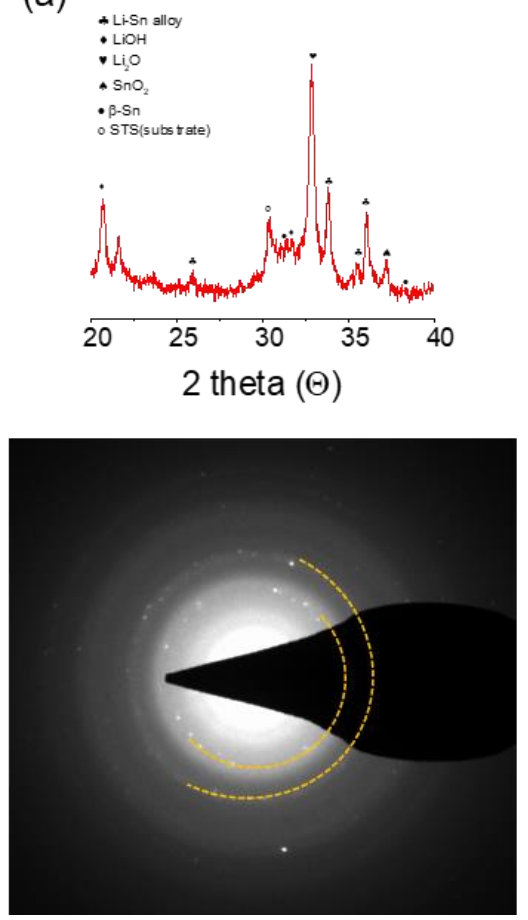

(b)
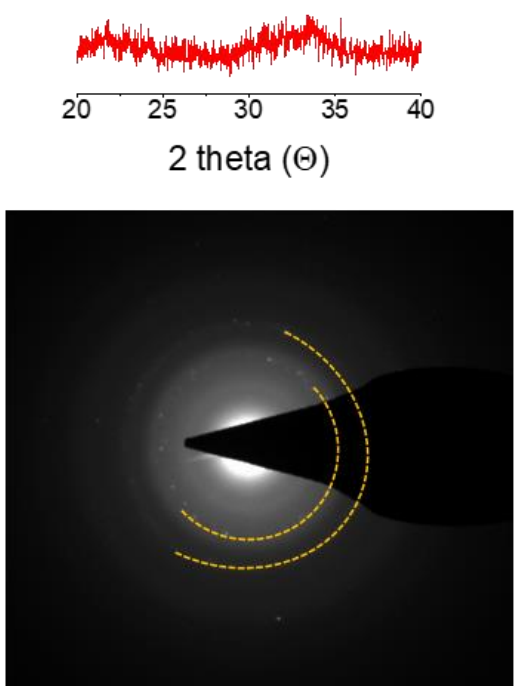

(c)
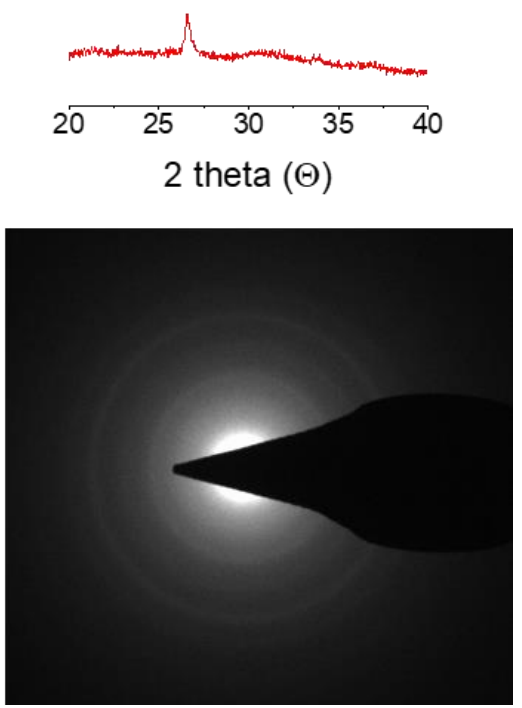

Fig. S10. Post-mortem analysis of cycled various particle size of $\mathrm{SnO}_{2}$-based anode through XRD and SAED pattern. a) $3 \mathrm{~nm}$, b) $10 \mathrm{~nm}$, and c) $100 \mathrm{~nm}$.
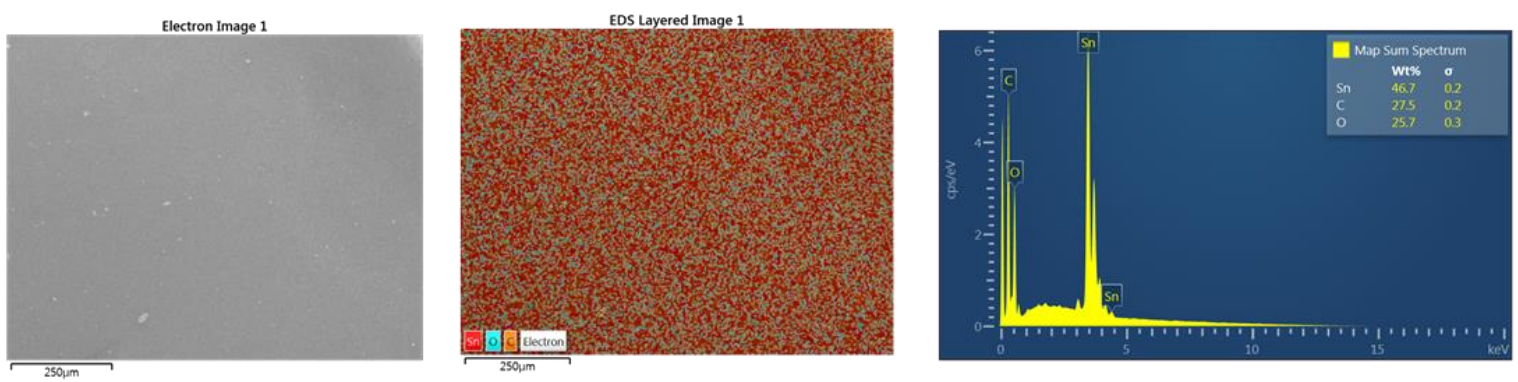

Fig. S11. EDS mapping image for quantum size of $\mathrm{SnO}_{2}$. 
Table S1. Quantitaive values depicted in Fig. $1 \mathrm{~b}$ derived from various $\mathrm{SnO}_{2}$ anodes.

\begin{tabular}{|c|c|c|c|c|c|c|}
\hline $\begin{array}{c}\text { Particle Size / } \\
\text { Parameters }\end{array}$ & $\begin{array}{c}\text { Power Density } \\
{\left[\mathbf{W} \mathbf{~ k g}^{-1}\right]}\end{array}$ & $\begin{array}{c}\text { Energy Density } \\
{\left[\mathbf{W h} \mathbf{~ k g}^{-\mathbf{1}}\right]}\end{array}$ & $\begin{array}{c}\text { Activity } \\
{[\mathbf{\%}]}\end{array}$ & $\begin{array}{c}\mathbf{L i}^{+} \mathbf{d i f f u s i o n} \\
{\left[\mathbf{e}^{-\mathbf{1 0}} \mathbf{c m}^{\mathbf{2}} \mathbf{~ s}^{-\mathbf{1}}\right]}\end{array}$ & $\begin{array}{c}\mathbf{R}_{\text {SEI }} \\
{[\mathbf{\Omega}]}\end{array}$ & $\begin{array}{c}\text { Tortuosity } \\
{[\%]}\end{array}$ \\
\hline $3 \mathrm{~nm}$ & 13110 & 4.4625 & $70 \sim 100$ & 2.8 & 0.05 & $2.07 \mathrm{E}^{-07}$ \\
\hline $10 \mathrm{~nm}$ & 10210 & 1.73 & $27 \sim 97$ & 1.98 & 0.14 & $2.16 \mathrm{E}^{-06}$ \\
\hline $100 \mathrm{~nm}$ & 6550 & 0.94 & $2 \sim 19$ & 0.84 & 0.27 & $8.29 \mathrm{E}^{-06}$ \\
\hline $200 \mathrm{~nm}$ & 6390 & 0.42 & $1 \sim 10$ & 0.66 & 0.17 & $9.18 \mathrm{E}^{-06}$ \\
\hline
\end{tabular}

Table S2. EIS fitted parameters for $\mathrm{SnO}_{2}$-based anode using different active nanoparticles.

\begin{tabular}{|c|c|c|c|c|c|c|c|}
\hline Particle Size & $R_{\text {s }}(\Omega)$ & $R_{\text {SEl }}(\Omega)$ & CPESEI $\left(\mathrm{YO},\left(\mathrm{S}_{\mathrm{s}} \mathrm{cm}^{-2}\right)\right.$ & $\mathrm{R}_{\mathrm{CT}}(\Omega)$ & $\mathrm{CPECT}\left(\mathrm{YO},\left(\mathrm{S}_{\mathrm{s}} \mathrm{cm}^{-2}\right)\right.$ & $\mathrm{W}\left(\mathrm{YO},\left(\mathrm{S}_{\mathrm{scm}}{ }^{-2}\right)\right.$ & $\mathrm{DLi}^{+}\left(\mathrm{cm}^{2} \mathrm{~s}^{-1}\right)$ \\
\hline $3 \mathrm{~nm}$ & 2.35 & 0.05 & $1.97 \times 10^{-6}$ & 50.05 & $5.95 \times 10^{-6}$ & $1.49 \times 10^{-3}$ & $2.8 \times 10^{-10}$ \\
\hline $10 \mathrm{~nm}$ & 4.57 & 0.14 & $4.35 \times 10^{-5}$ & 52.65 & $5.77 \times 10^{-6}$ & $1.44 \times 10^{-3}$ & $1.98 \times 10^{-10}$ \\
\hline $100 \mathrm{~nm}$ & 4.59 & 0.27 & $2.43 \times 10^{-6}$ & 70.33 & $1.32 \times 10^{6}$ & $1.41 \times 10^{-3}$ & $0.84 \times 10^{-10}$ \\
\hline $200 \mathrm{~nm}$ & 3.16 & 0.17 & $3.30 \times 10^{-6}$ & 78.29 & $1.34 \times 10^{-3}$ & $2.23 \times 10^{-3}$ & $0.66 \times 10^{-10}$ \\
\hline
\end{tabular}


Table S3. Comparison of electrochemical performance of previously reported $\mathrm{SnO}_{2}$ based anodes for Li-ion batteries.

\begin{tabular}{|c|c|c|c|c|c|c|c|c|c|}
\hline $\mathrm{SnO}_{2}$ (content) & Morphology & Particle Size \& & bonding & Voltage window (V) & $\begin{array}{c}\text { Capacity }\left(\mathrm{mAh} \mathrm{g}^{-1}\right) \\
\text { (Current Density),Cycle }\end{array}$ & $\begin{array}{c}\text { Es } \\
\left(W^{-1} \mathrm{~kg}^{-1}\right)\end{array}$ & Cyclability (\%) & and condition & Ref. \\
\hline $\mathrm{CNT}-\mathrm{SnO}_{2}$ & particle & $3 \mathrm{~nm}$ & Sn-C & $0.05-2.5$ & $1785\left(0.875 \mathrm{~A} \mathrm{~g}^{-1}\right), 500$ & 4462.5 & $10000(468 \%)$ & $17.5 \mathrm{~A} \mathrm{~g}^{-1}$ & Our Work \\
\hline $\mathrm{Sb}$-doped $\mathrm{SnO}_{2} /$ graphene-CNT & particle & $4-5 \mathrm{~nm}$ & $\mathrm{O}-\mathrm{C}=\mathrm{O}$ & $0.005-3.0$ & $983\left(1 \mathrm{~A} \mathrm{~g}^{-1}\right), 200$ & 2949 & $1000(73 \%)$ & $1 \mathrm{Ag}^{-1}$ & S1 \\
\hline CNT@void@SnO $2 @ C$ & particle & $100-150 \mathrm{~nm}$ & $\mathrm{O}-\mathrm{C}=\mathrm{O}$ & $0.01-3.0$ & $702.5\left(0.2 \mathrm{~A} \mathrm{~g}^{-1}\right), 350$ & 2107 & $350(74 \%)$ & $0.2 \mathrm{~A} \mathrm{~g}^{-1}$ & S2 \\
\hline CNT@SnO $2 @ \mathrm{C}$ & rod & N/A & N/A & $0.05-2.5$ & $984\left(0.72 \mathrm{~A} \mathrm{~g}^{-1}\right), 40$ & 2460 & $40(70 \%)$ & $0.72 \mathrm{~A} \mathrm{~g}^{-1}$ & S3 \\
\hline $\mathrm{Sn-SnO}{ }_{2} @ \mathrm{CNT}$ & particle & $<10 \mathrm{~nm}$ & $\mathrm{Sn}-\mathrm{O}-\mathrm{C}$ & $0.01-3.0$ & $1056\left(0.2 \mathrm{~A} \mathrm{~g}^{-1}\right), 200$ & 3168 & $1000(86 \%)$ & $0.5 \mathrm{~A} \mathrm{~g}^{-1}$ & S4 \\
\hline $\mathrm{SnO}_{2}-\mathrm{CNT}$ & particle & $3-5 \mathrm{~nm}$ & N/A & $0.005-2.0$ & $497\left(0.2 \mathrm{~A} \mathrm{~g}^{-1}\right), 300$ & 994 & $300(90 \%)$ & $0.2 \mathrm{~A} \mathrm{~g}^{-1}$ & S5 \\
\hline $\mathrm{SnO}_{2} @ \mathrm{lL}-\mathrm{CNTs}$ & particle & $3 \mathrm{~nm}$ & N/A & $0.01-3.0$ & $1438.1\left(0.5 \mathrm{~A} \mathrm{~g}^{-1}\right), 800$ & 4314 & $553(216 \%)$ & $0.5 \mathrm{Ag}^{-1}$ & S6 \\
\hline $\mathrm{SnO}_{2} @ \mathrm{PC}$ & particle & $100 \mathrm{~nm}$ & $\mathrm{~N} / \mathrm{A}$ & $0.01-3.0$ & $508\left(0.3 \mathrm{~A} \mathrm{~g}^{-1}\right), 300$ & 1524 & $300(63 \%)$ & $0.3 \mathrm{~A} \mathrm{~g}^{-1}$ & S7 \\
\hline $\mathrm{CNT} / \mathrm{c}-\mathrm{SnO}_{2}$ & particle & $70-80 \mathrm{~nm}$ & Sn-O-C & $0.01-3.0$ & $1140\left(0.05 \mathrm{~A} \mathrm{~g}^{-1}\right), 500$ & 3409 & $500(>72.0 \%)$ & $1.0 \mathrm{~A} \mathrm{~g}^{-1}$ & S8 \\
\hline Carbon coated-CNT Sponge/SnO & particle & $\sim 5 \mathrm{~nm}$ & N/A & $0.01-3.0$ & $\sim 943\left(0.1 \mathrm{~A} \mathrm{~g}^{-1}\right), 100$ & 2829 & $100(>90.56 \%)$ & $0.1 \mathrm{Ag}^{-1}$ & S9 \\
\hline $\mathrm{CNT@SnO} 2$ & particle & $3.7 \sim 5.9 \mathrm{~nm}$ & $\mathrm{Sn}-\mathrm{C}$ & $0.001-2.5$ & $\sim 682\left(0.05 \mathrm{~A} \mathrm{~g}^{-1}\right), 100$ & 1704 & $100(>10.1 \%)$ & $0.05 \mathrm{~A} \mathrm{~g}^{-1}$ & $\mathrm{~S} 10$ \\
\hline SWNT Paper/SnO ${ }_{2}$ & particle & $\sim 5 \mathrm{~nm}$ & N/A & $0.01-2.0$ & $\sim 669.52\left(0.025 \mathrm{~A} \mathrm{~g}^{-1}\right), 100$ & 1332 & $100(>67.8 \%)$ & $0.025 \mathrm{~A} \mathrm{~g}^{-1}$ & S11 \\
\hline $\mathrm{SnO}_{2}-\mathrm{Fe} 2 \mathrm{O} 3 / \mathrm{SWCNTS}$ & particle & N/A & N/A & $0.01-3.0$ & $692\left(0.2 \mathrm{~A} \mathrm{~g}^{-1}\right), 50$ & 2076 & $50(65 \%)$ & $0.2 \mathrm{~A} \mathrm{~g}^{-1}$ & $\mathrm{~S} 12$ \\
\hline Carbon/CNT/SnO 2 & particle & $7 \mathrm{~nm}$ & $\mathrm{O}-\mathrm{C}=\mathrm{O}$ & $0.01-2.5$ & $1572\left(0.2 \mathrm{~A} \mathrm{~g}^{-1}\right), 150$ & 3930 & $150(60.7 \%)$ & $1 \mathrm{Ag}^{-1}$ & $\mathrm{~S} 13$ \\
\hline $\mathrm{CNT} /$ Perforated $\mathrm{SnO}_{2}$ & particle & $0.6 \mu \mathrm{m}$ & $\mathrm{O}-\mathrm{C}=\mathrm{O}$ & $0.001-3.0$ & $1108\left(1.5 \mathrm{~A} \mathrm{~g}^{-1}\right), 1000$ & 3323 & $1000(>74.5 \%)$ & $1.5 \mathrm{~A} \mathrm{~g}^{-1}$ & S14 \\
\hline $\mathrm{SnO}_{2}$ nanoparticles/graphene & particle & $3-8 \mathrm{~nm}$ & N/A & $0.01-3.0$ & $978.6\left(0.2 \mathrm{~A} \mathrm{~g}^{-1}\right), 100$ & 2935.8 & $100(85.7 \%)$ & $0.2 \mathrm{Ag}^{-1}$ & S15 \\
\hline
\end{tabular}

Table S4. Deconvoluted peak area obtained from the C1s and O1s spectra through XPS measurement.

\begin{tabular}{|c|c|c|c|c|c|c|c|c|}
\hline Particle Size / Bonding & C-Sn & C=C & C-C & C-O & C=O & SnO2 & O & C-O \\
\hline $3 \mathrm{~nm}$ & 57325 & 122074 & 70793 & 36971 & 7396 & 170058 & 114326 & 86715 \\
\hline $10 \mathrm{~nm}$ & 16396 & 86989 & 25057 & 36323 & 14263 & 265567 & 131425 & 33558 \\
\hline $100 \mathrm{~nm}$ & 18711 & 89868 & 35413 & 32435 & 11706 & 235812 & 101132 & 61622 \\
\hline $200 \mathrm{~nm}$ & 23955 & 125919 & 32100 & 42199 & 17668 & 148912 & 55844 & 18776 \\
\hline
\end{tabular}




\section{Specific capacity Calculation}

The calculation for discharge capacity followed in this work is as follows,

$$
C_{D}=\Delta t \times I / m
$$

where, $\Delta \mathrm{t}$ is discharge time in hours, $\mathrm{I}$ is the current density in $\mathrm{mA} \mathrm{g}^{-1}$ and $\mathrm{m}$ is mass of the active material. ${ }^{\mathrm{S} 16}$ 


\section{REFERENCES}

[S1] Cui, J.; Yao, S.; Huang, J, -Q.; Qin, L.; Chong, W, G.; Sadighi, Z.; Huang, J.; Wang, Z.; Kim, J, -K. Sb-Doped $\mathrm{SnO}_{2} /$ Graphene-CNT Aerogels for High Performance Li-Ion and Na-Ion Battery Anodes. Energy Storage Materials 2017, 9, 85.

[S2] Tian, Q.; Tian, Y.; Zhang, Z.; Yang, L.; Hirano, S,-i. Fabrication of CNT@Void@ $\mathrm{SnO}_{2} @ \mathrm{C}$ with Tube-in-Tube Nanostructure as High-Performance Anode for LithiumIon Batteries. J. Power Sources 2015, 291, 173.

[S3] Chen, S.; Xin, Y.; Zhou, Y.; Zhang, F.; Ma,Y.; Zhou, H.; Qi, L. Branched CNT@SnO 2 Nanorods@Carbon Hierarchical Heterostructures for Lithium Ion Batteries with High Reversibility and Rate Capability. J. Mater. Chem. A 2014, 2, 15582.

[S4] Sun, L.; Si, H.; Zhang, Y.; Shi, Y.; Wang, K.; Liu, J.; Zhang, Y. Sn-SnO2 Hybrid Nanoclusters Embedded in Carbon Nanotubes with Enhanced Electrochemical Performance for Advanced Lithium Ion Batteries. J. Power Sources 2019, 415, 126.

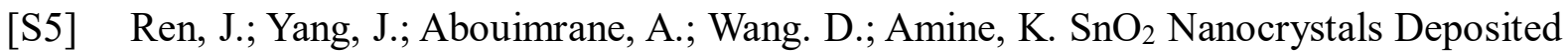
on Multiwalled Carbon Nanotubes with Superior Stability as Anode Material for Li-Ion Batteries. J. Power Sources 2011, 196, 8701.

[S6] Liu, K.; Zhu, S.; Dong, X.; Huang, H.; Qi, M. Ionic Liquid-Assisted Anchoring $\mathrm{SnO}_{2}$ Nanoparticles on Carbon Nanotubes as Highly Cyclable Anode of Lithium Ion Batteries. Adv. Mater. Interf. 2020, 7, 1901916.

[S7] Gong, K.; Ma, Y.; Zhang, T.; Yan, L.; Miao, Y.; Gao, F. In Situ Microwave Synthesis of $\mathrm{SnO}_{2}$-Porous Biomass Carbon as Anode Materials for Lithium-Ion Batteries. Adv. Eng. Mater. 2021, https://doi.org/10.1002/adem.202100064.

[S8] Bhattacharya, P.; Lee, J, H.; Kar, K, K.; Park, H, S. Carambola-Shaped $\mathrm{SnO}_{2}$ Wrapped 
in Carbon Nanotube Network for High Volumetric Capacity and Improved Rate and Cycle Stability of Lithium Ion Battery. Chem. Eng. J. 2019, 369, 422.

[S9] Luo, B.; Qiu, T.; Wang, B.; Hao, L.; Li, X.; Cao, A.; Zhi, L. Freestanding CarbonCoated CNT/Sn( $\left.\mathrm{O}_{2}\right)$ Coaxial Sponges with Enhanced Lithium-Ion Storage Capability. Nanoscale 2015, 7, 20380.

[S10] Cheng, Y.; Huang, J.; Qi, H.; Cao, L.; Yang, J.; Xi, Q.; Luo, X.; Yangisawa, K.; Li, J. Adjusting the Chemical Bonding of $\mathrm{SnO}_{2} @ \mathrm{CNT}$ Composite for Enhanced Conversion Reaction Kinetics. Small 2017, 13, 1700656.

[S11] Noerochim, L.; Wang, J,-Z.; Chou, S,-L.; Wexler, D.; Liu, H,-K. Free-Standing SingleWalled Carbon Nanotube/ $\mathrm{SnO}_{2}$ Anode Paper for Flexible Lithium-Ion Batteries. Carbon 2012, 50, 1289.

[S12] Wu, W.; Zhao, Y.; Li, J.; Wu, C.; Guan, L. A Ternary Phased $\mathrm{SnO}_{2}-\mathrm{Fe}_{2} \mathrm{O}_{3} / \mathrm{SWCNTs}$ Nanocomposite as a High Performance Anode Material for Lithium Ion Batteries. $J$. Energy Chem. 2014, 23, 376.

[S13] Ma, C.; Zhang, W.; He, Y,-S.; Gong, Q.; Che, H.; Ma, Z,-F. Carbon Coated $\mathrm{SnO}_{2}$ Nanoparticles Anchored on CNT as a Superior Anode Material for Lithium-Ion Batteries. Nanoscale 2016, 8, 4121.

[S14] Choi, S, H.; Lee, J,-H.; Kang, Y, C. Perforated Metal Oxide-Carbon Nanotube Composite Microspheres with Enhanced Lithium-Ion Storage Properties. ACS Nano 2015, 9, 10173.

[S15] Shi, S.; Deng, T.; Zhang, M.; Yang, G. Fast Facile Synthesis of $\mathrm{SnO}_{2} / \mathrm{Graphene}$ Composite Assisted by Microwave as Anode Material for Lithium-Ion Batteries. Electrochim. Acta 2017, 246, 1104.

[S16] Wang, H.; Liang, Y.; Gong, M.; Li, Y.; Chang, W.; Mefford, T.; Zhou, J. Wang, J.; 
Regier, T.; Wei, F.; Dai, H. An Ultrafast Ni-Fe Battery from Strongly Coupled Inorganic Nanoparticle/Nanocarbon Hybrid Materials. Nat. Commu. 2017, 3, 917. 